\title{
Analysis of Blocking Probability for Connection Management Schemes in Optical Networks
}

\author{
Jason P. Jue and Gaoxi Xiao \\ Center for Advanced Telecommunications Systems and Services \\ The University of Texas at Dallas \\ Richardson, TX 75083 \\ jjue@utdallas.edu,gxiao@utdallas.edu
}

\begin{abstract}
In this paper, we develop a model for evaluating the blocking probability of various connection management protocols for wavelengthrouted optical networks with dynamic lightpath establishment. The model characterizes both the blocking due to insufficient resources, as well as the blocking due to multiple interfering connection requests. We then use the analytical model to compare two connection management schemes, one which utilizes source-initiated reservation, and another which utilizes destination-initiated reservation.
\end{abstract}

\section{INTRODUCTION}

In a wavelength-routed optical network, all-optical connections, or lightpaths, may be established on an end-to-end basis between two nodes. These lightpaths provide transparent communications and eliminate electronic costs and bottlenecks at intermediate nodes. If the traffic in the wavelength-routed network is dynamic, then connection requests for establishing lightpaths will arrive to the network over time. When a connection request arrives to the network, a connection management protocol is responsible for finding a route and a wavelength for the lightpath, and for reserving the appropriate network resources. After some holding time, the connection may depart from the network and the lightpath will be removed.

A key measure of performance in dynamic wavelengthrouted networks is the blocking probability, or the probability that an incoming connection request will be denied. One source of connection blocking is insufficient network resources. If a route with sufficient capacity cannot be found between the source node and destination node, then the connection request must be blocked. Furthermore, if there are no wavelength converters in the network, then the lightpath for the connection must utilize the same wavelength on each link in the path between the source node and the destination node. If no such wavelength is available, then the connection will be blocked, even if capacity is available.

Connection blocking may also occur when routing and wavelength assignment decisions are made based on outdated network state information. State information may be outdated if state updates have not yet propagated throughout the network, or if multiple simultaneous connection requests interfere with each other. When a lightpath is being established, control messages must propagate along the route of the lightpath in order to reserve network resources. It is possible that resources along the route which were available when the connection re- quest was first initiated at the source node will no longer be available by the time the control message reaches the desired resource. In such a case, the request will be blocked.

While near-term emerging systems may be fairly static, with lightpaths being established for long periods of time, it is expected that, as network traffic continues to scale up and become more bursty in nature, a higher degree of multiplexing and flexibility will be required at the optical layer. Thus, lightpath establishment will become more dynamic in nature, with connection requests arriving at higher rates, and lightpaths being established for shorter time durations. In such situations, blocking due to conflicting connection requests may become an increasingly significant component of the overall connection blocking probability.

Blocking probability in wavelength-routed optical networks has been studied analytically in a number of previous works [1], [2], [3]; however, most prior studies focus on blocking due to insufficient network resources while ignoring the blocking caused by simultaneous connection requests.

In this paper, we develop an analytical model to investigate the blocking caused by insufficient resources, as well as blocking caused by interfering connection requests. We study the effect of both load and connection request arrival rate on the different types of blocking, and we compare different signaling and reservations protocols. The paper is organized as follows. In Section II, we present an overview of the network architecture and the and the signaling protocols for establishing lightpaths. Section III presents the analytical model for blocking probability. Section IV provides numerical examples, comparing the analysis with simulation results for a specific network topology, and Section V concludes the paper.

\section{PRotocols}

In this work, we consider a wavelength-routed optical network in which there are no wavelength converters. Connection requests arrive to the network dynamically, and for each connection request, a lightpath should be established. If a lightpath cannot be established, then the connection request is blocked.

Establishing a lightpath in a wavelength-routed network involves finding a route, assigning a wavelength, and reserving resources for the lightpath. The routing and wavelength assignment protocols can either rely on global state informa- 
tion, as in link-state routing, or they can rely on local information. It has been shown that protocols which utilize global state information generally outperform protocols in which no global information is available; however, global-informationbased schemes require a high degree of control overhead in order to maintain the state information. If the number of nodes and links in the network is large, or if connections are arriving and departing at a high rate, then it becomes increasingly difficult to maintain global information, and it becomes highly likely that some nodes will have outdated information. In such cases, it may be preferable to utilize protocols which do not rely on global information.

In this work, we assume shortest path routing; however, the analysis may be extended to any fixed routing scheme. Wavelength assignment is assumed to be random, with the wavelength being randomly selected with uniform distribution from among the set of feasible wavelengths along the route. The set of feasible wavelengths consists of the wavelengths which are available on all links in the route.

A signaling protocol is required to reserve resources along the selected route. In this paper, we investigate a sourceinitiated reservation protocol in which global information is available, and a destination-initiated reservation protocol in which only local information is available [4]. In sourceinitiated reservation protocols, the source node sends a control message towards the destination node along a selected route. The control message reserves resources on links as it travels towards the destination node. In destination-initiated reservation protocols, the source node sends a control message towards the destination node, but this control message does not reserve resources. Instead, the control message gathers wavelength availability information on its way to the destination. The destination, upon receiving the control message, selects a wavelength and sends its own control message back towards the source node along a specified route. This new control message attempts to reserve the selected wavelength along the links as it travels back towards the source node.

\section{AnAlyticAl MODEL}

We define the following parameters:

- $C$ : Number of wavelengths.

- $R$ : Set of routes.

- $l_{i}$ : The $i$ th link in a route $r \in R$.

- $\alpha^{r}$ : Arrival rate of connections to a route $r \in R$.

- $\alpha_{l_{i}}^{r}$ : Arrival rate of connections to route $r$ which are routed over link $l_{i}$.

- $\alpha_{l_{i}}$ : Arrival rate of connections to link $l_{i}$.

- $\frac{1}{\mu}$ : Mean holding time for a connection.

- $\tau_{l_{i}}^{r}$ : Propagation delay on link $l_{i}$ of route $r$.

- $t_{l_{i}}$ : Delay from time that link $l_{i}$ was last observed until the time that an attempt to reserve link $l_{i}$ is made.

\section{A. Blocking Due to Load}

In this section, we present an analytical model for the case in which links are assumed to be independent of each other, and wavelengths on a link are assumed to be independent of one another. This analysis is due to [1].

We consider the blocking on a route $r \in R$ which has $H$ hops. If the arrival rate of connection requests to each link is assumed to be a Poisson process with rate $\alpha_{l_{i}}$, and the connection holding time is exponentially distributed with mean $\frac{1}{\mu}$, then each link can be modeled as an $M / M / C / C$ queueing system. The probability that $n$ wavelengths are occupied on link $l_{i}$ is then given by:

$$
q_{l_{i}}(n)=\frac{\frac{1}{n !}\left(\frac{\alpha_{l_{i}}}{\mu}\right)^{n}}{\sum_{j=0}^{C} \frac{1}{j !}\left(\frac{\alpha_{l_{i}}}{\mu}\right)^{j}},
$$

and the probability that $n$ wavelengths are available on link $l_{i}$ is:

$$
p_{l_{i}}(n)=q_{l_{i}}(C-n) .
$$

A connection traversing $H$ hops will be blocked if there is no single wavelength which is available on all of the links in the path.

Let $a_{n}\left(w_{l_{1}}, w_{l_{2}}, \cdots, w_{l_{H}}\right)$ be the probability that $n$ wavelengths are available along the path, given that $w_{l_{i}}$ wavelengths are available on link $l_{i}$. The unconditional probability that $n$ wavelengths are available along a path $r$ is given by:

$$
\begin{aligned}
& P_{\text {avail }}^{r}(n)=\sum_{w_{l_{1}}=0}^{C} \sum_{w_{l_{2}}=0}^{C} \cdots \sum_{w_{l_{H}}=0}^{C} \\
& \quad p_{l_{1}}\left(w_{l_{1}}\right) p_{l_{2}}\left(w_{l_{2}}\right) \cdots p_{l_{H}}\left(w_{l_{h}}\right) a_{n}\left(w_{l_{1}}, w_{l_{2}}, \cdots, w_{l_{H}}\right)(3)
\end{aligned}
$$

The probability of a connection being blocked due to insufficient resources on a given route $r$ is then:

$$
P_{b l \_p a t h}^{r}=P_{a v a i l}^{r}(0)
$$

If there are two links in the path, then:

$$
\begin{aligned}
& a_{n}\left(w_{l_{1}}, w_{l_{2}}\right)= \\
& \left(\begin{array}{c}
w_{l_{2}} \\
n
\end{array}\right)\left(\prod_{i=1}^{n} \frac{w_{l_{1}}-i+1}{C-i+1}\right)\left(\prod_{i=1}^{w_{l_{2}}-n} \frac{C-w_{l_{1}}-i+1}{C-n-i+1}\right) \\
& \quad \text { if } n \leq w_{l_{1}}, w_{l_{2}} \leq C \text { and } w_{l_{1}}+w_{l_{2}}-n \leq C \\
& =1 \quad \text { if } n=0 \text { and either } w_{l_{1}}=0 \text { or } w_{l_{2}}=0 \\
& =0 \quad \text { otherwise }
\end{aligned}
$$

For an $H$ hop path,

$a_{n}\left(w_{l_{1}}, w_{l_{2}}, \cdots, w_{l_{H}}\right)=\sum_{k=n}^{w_{l_{H-1}}} a_{n}\left(k, w_{l_{H}}\right) a_{k}\left(w_{l_{1}}, \cdots, w_{l_{H-1}}\right)$ 


\section{B. Blocking Due to Dynamic Conflicts}

In a network with dynamic lightpath establishment, it is possible for connection requests to be blocked by other simultaneous connection requests. More generally, blocking may occur if the connection management entity has outdated information and makes routing or wavelength assignment decisions based on this outdated information.

We consider the dynamic situation in which global information is known at the time of the connection request, and is used to determine the route and wavelength for the connection. However, the network state may change as the connection is being established, possibly resulting in the connection being blocked. When the connection request first arrives to the source, the source node knows exactly which wavelengths are available along the entire path to the destination. If no wavelengths are available, the connection request is blocked immediately; otherwise, the source node chooses one of the available wavelengths and attempts to establish the lightpath on this wavelength. While the control message is propagating towards the destination, it is possible that a wavelength which was available on a link at the time that the connection request arrived has now been reserved by another connection request. In this situation, the original connection will be blocked. The total blocking probability for a connection on route $r$ is then:

$$
P_{b l o c k}^{r}=P_{b l_{-} p a t h}^{r}+\left(1-P_{b l_{-} p a t h}^{r}\right) P_{c o n f l i c t}^{r},
$$

where $P_{\text {conflict }}^{r}$ is the probability of blocking due to a conflicting connection request.

If a conflicting connection blocks the original connection on some link $l_{i}$, and the conflicting connection also shares link $l_{i+1}$ with the original connection, then the conflicting connection will also block the original connection on link $l_{i+1}$. The probability of blocking due to conflict is given by:

$$
\begin{aligned}
P_{\text {conflict }}^{r}= & 1-\left(P_{n b}^{l_{1}}\right) \cdot\left(P_{n b}^{l_{2}} \mid P_{n b}^{l_{1}}\right) \cdot\left(P_{n b}^{l_{3}} \mid P_{n b}^{l_{1}}, P_{n b}^{l_{2}}\right) \cdots \\
& \left(P_{n b}^{l_{H}} \mid P_{n b}^{l_{1}}, P_{n b}^{l_{2}}, \cdots, P_{n b}^{l_{H-1}}\right)
\end{aligned}
$$

where $P_{n b}^{l_{i}}$ is the probability that the wavelength chosen by the original connection is not blocked on link $l_{i}$.

If the original lightpath is on route $r$, and if an interfering lightpath on route $\hat{r}$ does not block the original lightpath on link $l_{i} \in r, \hat{r}$, then it won't block the original lightpath on any further links, $l_{i+1}, l_{i+2}, \ldots, l_{H}$.

$$
\begin{gathered}
\left(P_{n b}^{l_{i}} \mid P_{n b}^{l_{1}} P_{n b}^{l_{2}} \ldots P_{n b}^{l_{i-1}}\right)= \\
\prod_{\substack{\hat{r} \in R: \\
l_{i} \in \hat{r}, l_{1}, \cdots, l_{i-1} \notin \hat{r}}} \operatorname{Prob}\{\text { lightpaths on } \hat{r} \text { do not block }\} \\
\end{gathered}
$$

The conflicting lightpaths on route $\hat{r}$ will not block the original lightpath if there are no wavelengths available along route $\hat{r}$, or if there are wavelengths available along route $\hat{r}$, but the conflicting lightpaths choose a wavelength other than the wavelength chosen by the original lightpath. The conflicting lightpaths will choose a different wavelength if the wavelength chosen by the original lightpath is not among the available wavelengths along route $\hat{r}$, or if the wavelength chosen by the original lightpath is among the available wavelengths along route $\hat{r}$, but the conflicting lightpaths on route $\hat{r}$ do not choose the same wavelength as the original lightpath.

Conflicting lightpaths on route $\hat{r}$ can interfere with the original lightpath if they arrived to link $l_{i}$ during the time it took for the original lightpath's control message to reach link $l_{i}$. For simplicity, we assume that a conflicting lightpath arriving on route $\hat{r}$ will always be established as long as a wavelength is available on route $\hat{r}$. Thus, if there are $w$ wavelengths available on route $\hat{r}$, and $n<w$ conflicting lightpaths arrive to route $\hat{r}$, then the wavelength chosen by the original lightpath will not be chosen by the conflicting lightpaths with probability $\frac{w-n}{w}$, assuming that the conflicting lightpaths choose a wavelength randomly. On the other hand, if $n \geq w$ conflicting lightpaths arrive to route $\hat{r}$, then the wavelength chosen by the original lightpath will always be chosen by at least one of the conflicting lightpaths on route $\hat{r}$.

$$
\begin{aligned}
& \left(P_{n b}^{l_{i}} \mid P_{n b}^{l_{1}} P_{n b}^{l_{2}} \ldots P_{n b}^{l_{i-1}}\right)= \\
& \prod_{\substack{\hat{r} \in R: \\
l_{i} \in \hat{r}, l_{1}, \ldots, l_{i-1} \notin \hat{r}}}\left[P_{\text {avail }}^{\hat{r}}(0)+\sum_{w=1}^{C} P_{\text {avail }}^{\hat{r}}(w) \cdot\right. \\
& \left.\left(\frac{C-w}{C}+\frac{w}{C} \sum_{n=0}^{w-1} \frac{e^{-\alpha^{\hat{r}} t_{l_{i}}\left(\alpha^{\hat{r}} t_{l_{i}}\right)^{n}}}{n !} \cdot \frac{w-n}{w}\right)\right]
\end{aligned}
$$

For the source-initiated reservation scheme, we assume that the global information available to the source node is current when the connection request is sent. Thus, the time from the last observation of link $l_{i}$ until an attempt is made to reserve link $l_{i}$ is equal to the propagation delay from the source node to link $l_{i}$ on a route $r$. This time is given by $t_{l_{i}}=\sum_{j=1}^{i-1} \tau_{l_{j}}^{r}$.

It is also possible to model the situation in which the state information at the source node is incorrect when the connection request is sent. In this case, $t_{l_{i}}$ should also include the propagation delay between the source node and the entity sending the last state update as well as the time from the last received state update to the time that the connection request is sent. We do not consider this situation in the paper.

For a destination-initiated reservation scheme, the delay must be measured from the time that the link was observed when the control message propagated towards the destination to the time that the control message attempts to reserve the wavelength as the control message propagates back towards the source. Thus, $t_{l_{i}}=\sum_{j=i}^{H} \tau_{l_{j}}^{r}+\sum_{k=i+1}^{H} \tau_{l_{k}}^{r}$.

Note that on the links shared by routes $\hat{r}$ and $r$, we know that the wavelength chosen by the original connection is available, otherwise the original connection would have been blocked. 
For example, if link $l_{i}$ is on both route $\hat{r}$ and $r$, and if the original connection chooses wavelength $\lambda_{1}$, then wavelength $\lambda_{1}$ is known to be available on link $l_{i}$. However, for simplicity of analysis, we do not take this wavelength's availability on the shared links into consideration when calculating the probabilities $P_{\text {avail }}^{\hat{r}}(n)$ on the interfering lightpath's route.

Another simplifying assumption in the analysis is that a conflicting lightpath which arrives and chooses the same wavelength as the orignial connection will remain long enough to block the original connection, i.e., the conflicting lightpath will not depart before the original lightpath's connection request message reaches the link. With this assumption, the analysis will tend to over-estimate the blocking probability, particularly for very short connection holding times.

\section{NuMERICAL EXAMPLES}

In this section, we compare two reservations/wavelength assignment schemes, a source-initiated reservation scheme in which global information is available, and a destinationinitiated reservation scheme in which no global information is available. Routing in either case is assumed to be fixed shortest path. For the source-initiated reservation scheme, it is assumed that the state information available to each node is up-to-date at the time that the connection request is initiated, and the wavelength selection is made at the source node based on this information.

We study the effect of simultaneous connection requests on blocking in the network shown in Fig. 1. The link distances are given in units of kilometers, and each link in the network can carry eight wavelengths. Connection requests arrive to the network according to a Poisson process with rate $\lambda$ connections per second, and connection holding times are exponentially distributed with an average holding time of $\frac{1}{\mu}$ seconds.

In Fig. 2, we examine the blocking probability of both the source-initiated reservation scheme and the destinationinitiated reservation scheme as a function of load $\left(\frac{\lambda}{\mu}\right)$. The average holding time is kept constant at $100 \mathrm{~ms}$. The results show that the source-initiated reservation scheme in which global link state information is available at the source node outperforms the destination-initiated scheme in which link state information must be collected as the control message heads towards the destination. The primary reason for this difference in performance is that, in the destination-initiated scheme, the time between the observation of a link and the attempt to reserve a wavelength on the link is, on average, twice as high as that for the source-initiated reservation scheme. This longer time until reservation leads to a greater probability that another connection will interfere and cause blocking.

We note that the analysis is fairly accurate for the source-initiated reservation scheme, but less accurate for the destination-initiated scheme. In the analysis, the blocking is calculated in two steps. The blocking due to load is calculated first, and this result is used to calculate the blocking due to conflicts. However, when connections are blocked due to conflicts, the overall load to the system will be reduced, leading to a reduction in the blocking due to load. Thus, a more accurate analysis would involve iteratively calculating the blocking probabilities over several steps. Since the destination-initiated reservation scheme has a greater amount of blocking due to conflict, the inaccuracy of the analysis due to this effect is greater than that for source-initiated reservation.

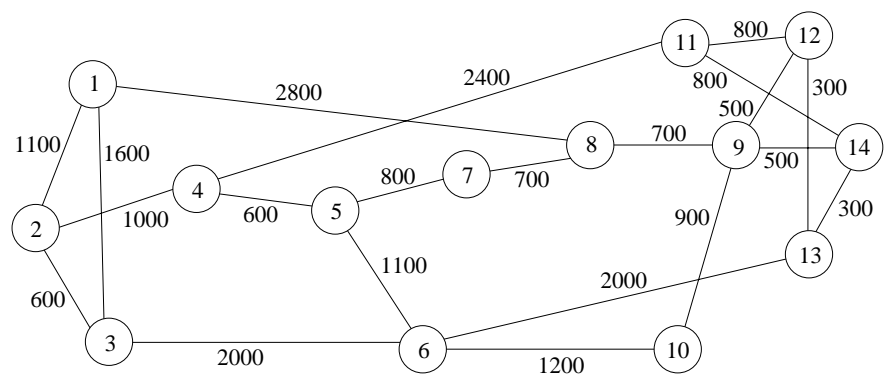

Fig. 1. Network Topology.

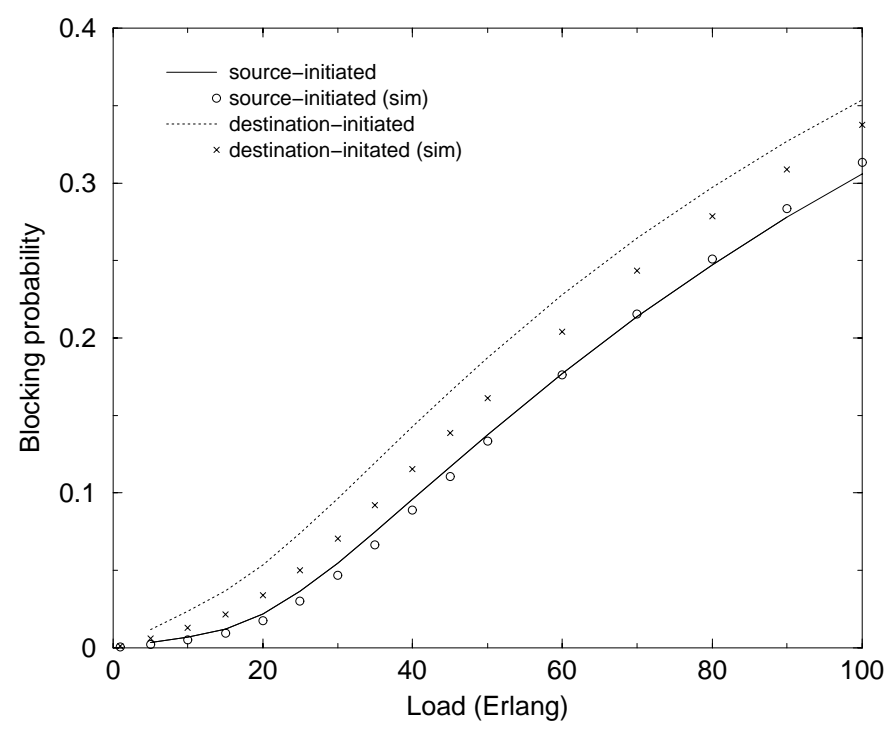

Fig. 2. Blocking probability versus load. $\frac{1}{\mu}=100 \mathrm{~ms}$.

Figure 3 shows the blocking due to load and the total blocking as a function of arrival rate. The overall load is kept constant at either 20 or 60 Erlangs by varying the average connection holding time along with the arrival rate.

At low arrival rates, blocking is caused primarily by the load on the network; however, as the arrival rate increases while the offered load remains constant, the blocking due to load doesn't increase by much. However, the blocking due to conflicting connection requests increases as expected and becomes the dominant source of blocking for networks with low loads.

As mentioned, the blocking due to load is overestimated by the analysis. In this figure, we observe that the reduction in load due to conflict blocking has a greater effect when the overall load is higher, thus the analysis for blocking due to load is less 


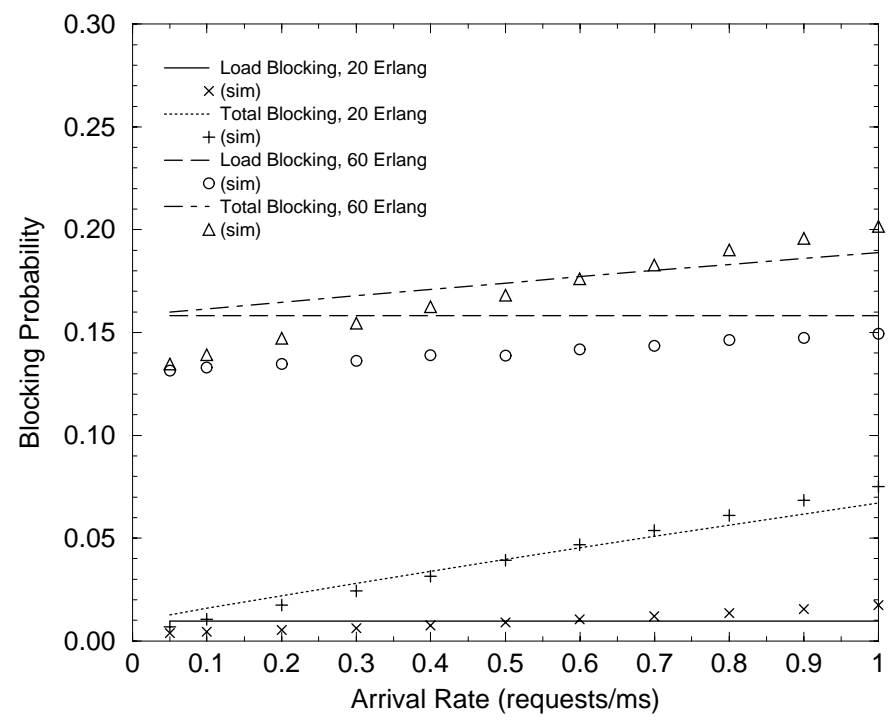

Fig. 3. Blocking probability versus arrival rate for source-initiated reservation scheme.

accurate for higher network loads.

We also observe that, in the analysis, the blocking due to load doesn't change as the arrival rate increases, whereas in the simulation, the blocking due to load increases slightly as the arrival rate increases. Although the offered load remains constant, the actual network utilization is increasing, since connections which are blocked still reserve network resources for a short period of time, leading to a slight increase in blocking probability. Furthermore, during the connection setup process when resources are being reserved, the reserved resources will go unused for a short period of time before the connection can begin transmitting data. This resource reservation overhead will be higher when the connections are established for a shorter time duration, and the number of connections being established is higher. Thus, as the arrival rate increases and the connection holding time decreases, the overall load in the network will tend to increase.

Figure 4 shows the blocking as a function of arrival rate for the destination-initiated reservation scheme. An interesting effect captured by the simulation is that, for higher loads, the blocking due to load decreases slightly as the arrival rate increases. A possible reason for this behavior is that, as a greater number of connections are blocked due to connection request conflicts, the actual load on the network decreases, and this decrease in the load is more significant that the increase in load caused by the additional resources being reserved by connections which are eventually blocked.

\section{CONCLUSION}

In this paper, we presented a model for evaluating the blocking probability in wavelength-routed optical networks with dynamic lightpath establishment. The model improves on existing models by considering blocking due to load as well as

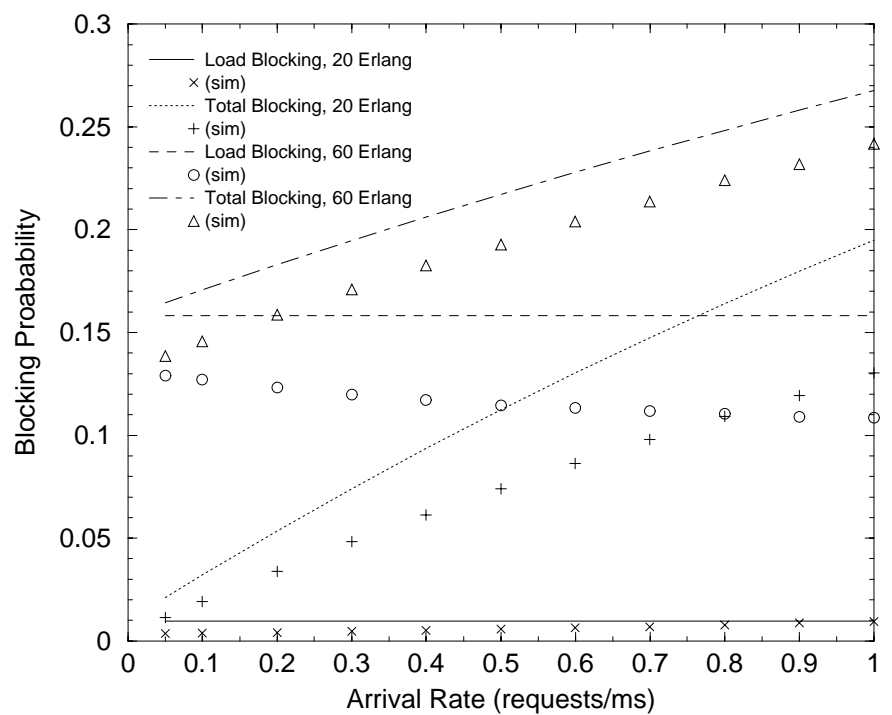

Fig. 4. Blocking probability versus arrival rate for destination-initiated reservation scheme.

blocking due to conflicts between multiple connection requests. The improved model enables the evaluation and comparison of various signaling and reservation schemes for establishing lightpaths in optical networks, and illustrates how the behavior of these schemes is affected by the connection arrival rate.

The analysis is applied to two reservation schemes, and it is shown that a source-initiated reservation scheme in which global information is available outperforms a destinationinitiated reservation scheme. The trade-off is that the destination-initiated reservation scheme does not need to maintain global state information, leading to significantly less control overhead.

Although the model is shown to be fairly accurate under certain conditions, there are still areas in which the model can be improved. In particular, the reduced load due to conflict blocking and the increased load due to reservation of resources by connections which are eventually blocked should be taken into consideration. The model may also be extended to consider a wider range of connection management schemes, such as the case in which source-initiated reservation is implemented without global state information.

\section{REFERENCES}

[1] A. Birman, "Computing Approximate Blocking Probabilities for a Class of All-Optical Networks," IEEE Journal on Selected Areas in Communications, vol. 14, no. 5, pp. 852-857, June 1996.

[2] R.A. Barry and P.A. Humblet, "Models of Blocking Probability in AllOptical Networks with and Without Wavelength Changers," IEEE Journal on Selected Areas in Communications, vol. 14, no. 5, pp. 858-867, June 1996.

[3] A. Mokhtar and M. Azizoglu, "Adaptive Wavelength Routing in AllOptical Networks," IEEE/ACM Transactions on Networking vol. 6, no. 2, pp. 197-206, April 1998.

[4] X. Yuan, R. Melhem, R. Gupta, Y. Mei, and C. Qiao, "Distributed Control Protocols for Wavelength Reservation and Their Performance Evaluation," Photonic Network Communications, vol. 1, no. 3, pp. 207-218, 1999. 